\title{
STUDY IN ESTIMATION OF COURSE TEACHING PLAN
}

\author{
Beijing Polytechnic University
}

Huang. zhenkan

\section{ABSTRACT}

This article is dealing with the dialectical relation of mutual effect and interaction between "Learning" and "Teaching" according to the quantitative analysis. We decide the procedure of teaching knowledge by using the method of Analytic Hierarchy Process. With the Analytic Process method we estimate the First value of fundamental knowledge given and Dynamic process to denote the weight Function of fundamental knowledge. The fundamental knowledge may be divided into two parts, that is new knowledge and memory one. According to the rule of teaching and psychological equation. we establish the estimation of the teaching plan and control of mathematical model.

select some proper state variable and control variable, using the method of dynamic programme to get the best optimal policy of teaching and control the best quanticy of teaching.

USE AHP METHOD TO DETERMINE THE CONTENT OF TEACHING KNOWLEDGE

For the purpose of having a definite object in view, first we should know what is the content of both new and old knowledge for the learners thus we promote the initiative of teaching activities, then we determine the weight function.

Suppose: 1. The content of teaching based on different sorts of basic knowledge separately.

2. These basic knowledge are belonging to $m$ periods (or some chapters).

First determine the weight function in $z$, then the $n$ in $m$, at last we calculate the weight function of $n$ in $z$. Use comparative scale put forward by saty to form contrast matrix.

In general, the procedure of teaching may be divided into three levels: Firstly. the most excellent plan of knowledge construction. We make as $w$.

Secondly, to divide the course periods make as $u=\left(u_{1} u_{2} \ldots, u\right)$

Thirdly, fundamental knowledge make as $w=\left(w_{1} \cdot w_{2} \cdot w_{n}\right)$

The relation between teaching course and time is very important. So the weight value of propriety order is the function of time. The weight value of basic knowledge. 


$$
w(t)=\sum_{i=1}^{n} w(t)
$$

In $w(t)(i=1,2, \ldots, n)$, the component of weioht function may be determined by the Dynamic propriety order method. In particular case when $t=0$

starting value: $\quad w(0)=\sum_{i=1}^{n} w_{i}(0)$.

In $w(0)(i=1,2, \ldots, n)$ determined by the prioriety order of $i$

state.

For example. according to the sampled distribution in mathematical statistics, the fundamental knowledge consists of Higher mathematics, probability, sampled distribution.

The first judgment matrix when $A(0)$

$$
A(0)=\left\{\begin{array}{ccc|}
1 & 4 / 3 & 3 \\
3 / 4 & 1 & 3 \\
1 / 3 & 1 / 3 & 1
\end{array}\right.
$$

And related the main latent root and latent rector separately are: $(0)=3.02, w(0)=(0.46,0.41,0.13)^{\mathrm{T}}$ Dynamistic proprietv relative weights of objects matrix $A(t)$

$A(t)=\left\{\begin{array}{cccc}1 & 1 & 5 & 1 \\ 1 & 5 & 5 & 0.1 t^{2}+0.8 t+0.2 \\ 0.1 t^{2}+0.8 t+0.2 & \ln (t+1)+0.4 & 1 & 1\end{array}\right.$

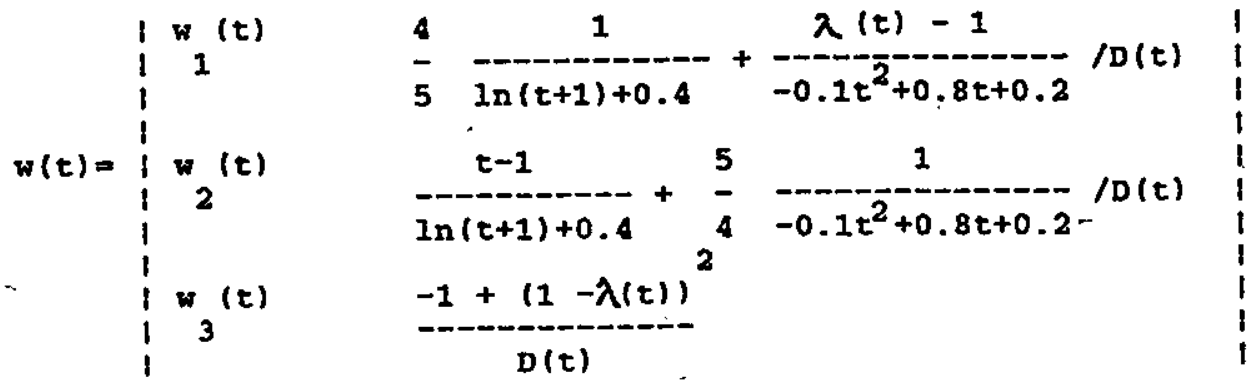


$D(t)=\frac{4}{5 \ln (t+1)+0.4}+\frac{\lambda(t)-1}{-0.1 t^{2}+0.8 t+0.2}+\frac{\lambda(t)-1}{\ln (t+1)+0.4}$

$$
+\frac{1}{4}-0.1 t^{2}+0.8 t+0.2-1+(1-\lambda(t))^{2}
$$

In $t ; w_{1}(t), w_{2}(t), w_{j}(t)$ are the weight function of Higher mathematics: Probabilicy and Sampled Distribution separately. See Figure $1 \mathrm{w}(t)$ and $w(t)$ are packing knowledge, $w(t)$ is new knowledge. 1

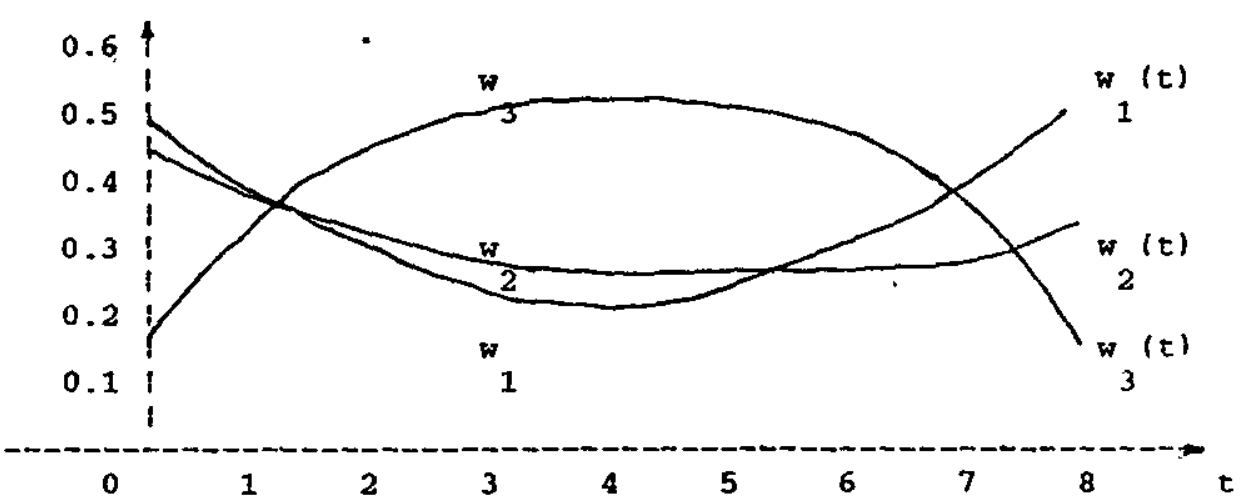

Figure 1 Weight Function of Fundamental knowledge.

THE ESTIMATION OF TEACHING PLAN AND CONTROLLED MATHEMATICAL MODEL

Teaching activities can be denoted by a psychological equation $i$. e. Response=sensitive $x$ stimulus. 1 . If stimulus is $t$, the density of taught knowledge would be at the density of students. learnt knowledge would be $y(t)$. The Density of the course itself will be $w(t)=(w(t), w(t), \ldots, w(t)) . w(0)=(w(0), w(0)$. $\ldots \ldots(0))$ is decided by (1) and $(2)^{n}$. n

2. The condition response of reaching and learning will be their alter rate $d x / d t$ and $d y / d t$ separately.

3. The sensitive factor is based on the ability factor of a certain teacher $\alpha=\left(\alpha_{1}, \alpha_{2}, \cdots \alpha_{n}\right)$ and it can be aot according to the estimation of teaching. $y=(\gamma ; \gamma, \ldots \gamma)$ is qotten by the students or strugaled for. students unacceptance rate is $=\alpha+\gamma-\alpha \gamma$.

From psychological equation we know:

$d x / d t=\alpha w(t)+\beta v(t)$ 


$$
\begin{aligned}
x(0) & =2 w(0) \\
\mathrm{dy} / \mathrm{d} t & =\gamma x(t)+\beta y(t) \\
y(0) & =y w(0)
\end{aligned}
$$

Suppose $T$ is the total sum of teaching hours: Definition:

The quanticy of teaching knowledqe $x(x(t))=\int_{0}^{T} x(t) d t$

The quantity of learning knowledge $F(y(t))=\int_{0}^{T} y(t) d t$

In studying the final control of teaching process, the variation problems hoping to let students get maximum quantity of knowledge, within the limited time $T$, and after the teacher has finished the quantity of knowledge $x$. $i$. e.

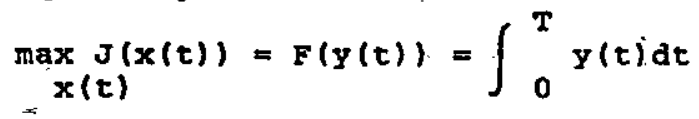

Constraint

$$
d y / d t=\gamma x(t)-\beta y(t) .
$$

Boundary condition

$$
\begin{gathered}
\int_{0}^{T} x(t) d t=x \\
d x / d t=\alpha w(t)+\beta y(t)
\end{gathered}
$$

starting condition

$$
\begin{aligned}
& x(0)=2 w(0) \\
& y(0)=\gamma w(0)
\end{aligned}
$$

Found $x(t)$, ' $t$ ' will be constraint by $(8)-(12)$ and (7) will be extremity. Introduce lagrange and multiply $\mu$. put (9) into objective function, the solution:

$$
\max _{x(t)} J(x(t))=F(y(t))+\mu \int_{0}^{T} x(t) d t
$$

8

and confined-into (8), (10), (11), (12).

DYNAMIC PROGRAMME PROBLEM

The teaching process of (13) may face following questions:

1. What is the starting point of the memory knowledoe by learning new knowledge?

2. According to the level of teaching of the teacher and quality of the learners to finish the teaching course, what is the quantity of students knowledge acquired.

3. How long is to finish the teaching course?

The above questions 'are closely related with one another and can't be considered separately. We should regard the whole teaching process as a special system and study it by Dynamic 
Programe, shown as Figure 2. For example, we divided the teaching time $T$ into four periods. Within $T$, the students of knowledge $F_{0}$. the quantity of acquired knowledqe will be $q_{1}(t)$ teaching plan in $x$, go on the procedure till finishing these four periods and at last we arrive at the whole quantity of teachina $X$ and total teaching time $\mathrm{T}$.

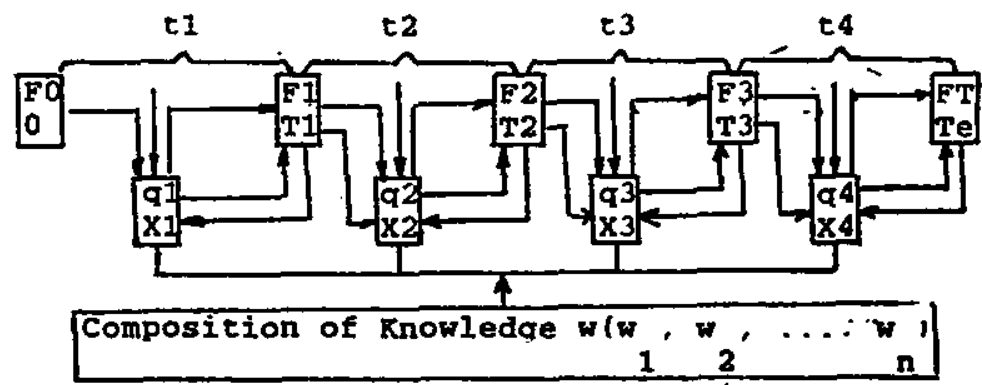

Figure 2 The Technological Process of Teaching and Learning

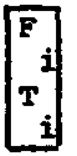
represents the state function of dynamic plan, that is:

period is state variable $F_{i}$, at the end of $i$. the quantity of knowledge (partial import of $i+1$ ) and accumulated time will be $T$ Peroid is import $F_{i-1}$ to its accumulateed time will be $T_{i-1} \cdot$ The square

$a$
if
$x$
i

is the state function of teachers determined variable. It is decided by variable values $a x$ of two states. $a$ is the tactics of teachina $x_{i}$ taught is the information we want

$T_{e}$ is a reasonable upper limit in fulfilling the process of the whole teaching. Use the princiole of obtimization (put into counter current and name the number). introduce (13) and aot relation formula, count the lenath of each periods time. teachina tactics, quantity of teachinq and learning. The calculation of others should be repeated till to satisfy $(9)$.

CONCLUSIONS

For the purpose of teachina effect wanted, we can adjust $w(t), \alpha$. $\boldsymbol{\gamma}$, and adopting some measures oudht to be concerned. At the 
same time the article can also introduce the way of stimulating activity of the participants, promoting the quality of teachinf and give the enlightment of scientific method of teaching and management of teaching.

\section{REFERENCES}

Saaty, T L. (1982), The Logic of Priorities. Kluwer. Nijhoff publishing pp 20-37, pp172-177

saaty, T. L. (1985) Analytical Planning, Peraamon Press, pp75-79 